\title{
Evaluation of circulating cell-free DNA as a molecular monitoring tool in patients with metastatic cancer
}

\author{
CLEMENS HUFNAGL $^{1 *}$, MICHAEL LEISCH ${ }^{2-4 *}$, LUKAS WEISS $^{2-4}$, THOMAS MELCHARDT $^{2-4}$, \\ MARTIN MOIK ${ }^{2-4}$, DANIELA ASSLABER ${ }^{2-4}$, GEISBERGER ROLAND ${ }^{2-4}$, PHILIPP STEININGER ${ }^{5}$, \\ THOMAS MEISSNITZER ${ }^{6}$, DANIEL NEUREITER ${ }^{1}$, RICHARD GREIL $^{2-4}$ and ALEXANDER EGLE ${ }^{2-4}$
}

\author{
${ }^{1}$ Institute of Pathology, Paracelsus Medical University Salzburg; ${ }^{2}$ IIIrd Medical Department with Hematology and \\ Medical Oncology, Oncologic Center, Paracelsus Medical University Salzburg; ${ }^{3}$ Salzburg Cancer Research Institute with \\ Laboratory of Immunological and Molecular Cancer Research and Center for Clinical Cancer and Immunology Trials; \\ ${ }^{4}$ Cancer Cluster Salzburg; ${ }^{5}$ Institute for Research and Development on Advanced Radiation Technologies, and \\ ${ }^{6}$ Institute of Radiology, Paracelsus Medical University Salzburg, A-5020 Salzburg, Austria
}

Received January 28, 2019; Accepted August 6, 2019

DOI: $10.3892 / 01.2019 .11192$

\begin{abstract}
The clinical decisions made when treating patients with metastatic cancer require knowledge of the current tumor extent and response to therapy. For the majority of solid tumors, a response assessment, which is based on imaging, is used to guide these decisions. However, measuring serum protein biomarkers (i.e. tumor markers) may be of additional use. Furthermore, tumor markers exhibit variable specificity and sensitivity and cannot therefore be solely relied upon when making decisions regarding cancer treatment. Therefore, there is a clinical requirement for the identification of specific, sensitive and quantitative biomarkers. In recent years, circulating cell-free DNA (cfDNA) and mutation-specific circulating cell-free tumor DNA (cftDNA) have been identified as novel potential biomarkers. In the current study, cfDNA and cftDNA were compared using imaging-based staging and current tumor markers in 15 patients with metastatic colorectal, pancreatic or breast cancer. These patients were treated at the
\end{abstract}

Correspondence to: Dr Alexander Egle, IIIrd Medical Department with Hematology and Medical Oncology, Oncologic Center, Paracelsus Medical University Salzburg, Müllner-Hauptstrasse 48, A-5020 Salzburg, Austria

E-mail: a.egle@salk.at

${ }^{*}$ Contributed equally

Abbreviations: cfDNA, circulating cell-free DNA; cftDNA, circulating cell-free tumor DNA; PET, Positron Emission Tomography; FFPE, formalin fixed with paraffin embedded; CEA, Carcinoembryonic antigen; CA15-3, Carcinoma Antigen 15-3; CA19-9, Carcinoma Antigen 19-9

Key words: liquid biopsy, circulating cell-free DNA, circulating cell-free tumor DNA, tumor burden, serum tumor markers, tumor-volumetry
Third Medical Department of Paracelsus Medical University Salzburg (Austria). The results of the current study demonstrated a statistically significant correlation between the concentration changes of cfDNA and cftDNA and response to treatment, which was assessed by imaging. A correlation was not indicated with current clinically used tumor markers, including carcinoembryonic antigen, carcinoma antigen 15-3 and carcinoma antigen 19-9. The present study also indicated a correlation between cfDNA and cftDNA and the tumor volume of metastatic lesions, which was not observed with the current clinically used tumor markers. In conclusion, cfDNA and cftDNA exhibit the potential to become novel biomarkers for the response assessment following cancer treatment, and may serve as a tool for the estimation of tumor volume. The current study further supports the increasingly important role of cfDNA and cftDNA as new monitoring tools for use during cancer therapy.

\section{Introduction}

Recurrent gene mutations are found in the majority of cancer types. Genotyping tumor tissue for somatic genetic alterations, which leads to an accurate diagnosis of the tumor type, guides treatment decisions and/or predicts the response to therapy, has become common practice in medical oncology (1-3). Currently, tissue samples obtained via surgery or biopsy are the gold standard for use in this analysis.

For the treatment of patients with metastatic cancer, knowledge of tumor mass dynamics and response to therapy are important. Currently, imaging techniques, including CT and positron emission tomography (PET)-CT scanning are most commonly used for this purpose (4-7). Additionally, serum protein biomarkers, which are often referred to as tumor markers, are used in clinical practice to assess tumor dynamics and treatment response over time $(8,9)$. However, the currently used tumor markers do not always accurately reflect the actual disease burden, and false positive results are sometimes seen in benign conditions such as inflammation $(10,11)$. Therefore, 
these markers cannot be solely relied upon when estimating actual tumor mass, and need to be interpreted together with imaging results $(12,13)$. Furthermore, for a number of tumor types, no reliable serum tumor marker has been identified.

There is an urgent clinical requirement for the identification of reliable tumor-specific biomarkers for the management of patients with metastatic cancer due to a number of reasons: i) Repetitive imaging studies can lead to a relevant radiation exposure; ii) the differentiation between residual viable tumor tissue and fibrotic tissue following neoadjuvant chemo- and/or radiationtherapy is often challenging, and iii) the differentiation between actual tumor progression and pseudoprogression, using current immunooncological approaches, can also be challenging in daily clinical practice (14).

In recent years, circulating cell-free DNA (cfDNA) has been indicated as a potential novel biomarker, largely due to the progression of sequencing technologies, including next generation sequencing and digital PCR (15). It has also been indicated that fragments of 'normal' DNA and circulating cell-free tumor DNA (cftDNA) enter the bloodstream via tumor-cells (16-18), cells undergoing apoptosis or necrosis or via the active release of DNA (19). Cell-free DNA can be detected in small amounts in healthy human plasma $(3,20,21)$. However, higher concentrations of cfDNA are detected in the plasma of patients with cancer, due to tumor cell necrosis, apoptosis or its active release by tumor cells $(3,22-24)$. cftDNA can reflect the mutations located in the primary tumor, including oncogene or tumor-suppressor gene mutations or gene-rearrangements $(21,25,26)$, and can potentially be used to predict tumor burden more accurately than the protein biomarkers currently used (8,27-29).

The purpose of the current study was to evaluate cfDNA and cftDNA and the correlation with serum protein tumor markers and imaging results during therapy of patients with metastatic colorectal cancer (CRC), pancreatic cancer (PC) or breast cancer $(\mathrm{BC})$.

\section{Materials and methods}

Patients and sample acquisition. The current study was approved by the Local Institutional Research Ethics Committee (415-E/1469/11-2013) and all patients provided written informed consent prior to blood sampling and tumor tissue analysis. Formalin-fixed paraffin-embedded (FFPE) tissue samples were obtained during surgery and analyzed at the Institute of Pathology, Paracelsus Medical University (Salzburg).

Blood sampling was performed between April 2012 and December 2013. Plasma samples were prospectively collected from 15 patients who were diagnosed and treated at the Department of Internal Medicine III, Salzburg Cancer Research Institute, Paracelsus Medical University Salzburg (Salzburg, Austria).

Patients were recruited consecutively within the study period. The only inclusion criterion was the diagnosis of metastatic CRC, PC or BC. Patients were considered for analysis if they had an adequate amount of sampling time-points available. All patients received at least one course of palliative systemic therapy. Patients with CRC most commonly received 5 -FU based regimens in combination with Oxaliplatin or
Irinotecan. Patients with PC were most commonly treated with Gemcitabine based regimens and patients with $\mathrm{BC}$ were mainly treated with Taxans (Paclitaxel or Docetaxel).

The response to treatment was assessed using CT scans that were performed at 8-12 weeks intervals as indicated by the physician. The response was defined according to the Response Evaluation Criteria in Solid Tumors (RECIST) (30-33).

Isolation of DNA from FFPE tissues. Genomic DNA was extracted from 3-7 sequential sections $(10 \mu \mathrm{m})$ of the primary tumor FFPE specimens. A Proteinase $\mathrm{K}$ tissue digestion was performed in a $1.5 \mathrm{ml}$ micro centrifuge tube containing 3-7 sections of paraffin-embedded tissue, and incubated at $70^{\circ} \mathrm{C}$ overnight to dissolve the tissue. DNA was then extracted using a Maxwell DNA LEV tissue DNA kit (Promega Corporation), according to the manufacturer's protocol, and eluted using $50 \mu 1$ elution buffer. The Maxwell ${ }^{\circledR} 16$ Instrument purifies DNA using silica-clad paramagnetic particles, which provide a mobile solid phase that optimizes the capture, washing and elution of the target material. The quality of extracted DNA was examined using agarose gel electrophoresis and ethidium bromide staining, and concentrations were evaluated using photometry (NanoDrop 1000 Spectrophotometers; Thermo Scientific Inc.).

Direct sequencing of FFPE samples. Primary tumor samples were analysed using PCR amplification and Sanger sequencing. A number of genes were analysed, including KRAS, NRAS, BRAF, Tp53, NOTCH, EGFR, PTEN and PI3K, which are commonly mutated in cancer (34-37). BigDye ${ }^{\circledR}$ Sequencing Master Mix was used to perform the sequencing reaction according to the manufacturer's protocol. The samples were analysed on a capillary sequencer ABI 3100-Analyser (Applied Biosystems; Thermo Fisher Scientific, Inc.). Oncogenic mutations were identified in all 15 FFPE samples.

Plasma samples and DNA purification. Serial blood samples (8 $\mathrm{ml}$ each) were collected upon and following diagnosis. Mandatory blood sampling was performed on all patients at staging time-points and at intervals of 1-3 weeks between these points (depending on the frequency of clinical visits). All samples were processed within 30 min following blood collection and centrifuged once for $10 \mathrm{~min}$ at $1,500 \mathrm{x} \mathrm{g}$. The resulting plasma sample was spun once more for $10 \mathrm{~min}$ at high speed $(2,000 \times \mathrm{g})$ in order to purify plasma from any remaining blood cells. The plasma was aliquoted and stored at $-80^{\circ} \mathrm{C}$.

Total nucleic acids were purified from $1 \mathrm{ml}$ plasma using a modified phenol-chloroform extraction method, as previously described (38). A total of 108 serial plasma samples were obtained from 15 patients.

Identification of cfDNA somatic alterations in plasma. The specific mutations indicated in the primary analysis of FFPE samples (using Sanger sequencing) were used for every specific patient as a target for ultra-deep cfDNA sequencing.

By designing sequences that flank the target regions of interest, the specific PCR-products for ultra-deep sequencing were isolated. This process was used to prepare libraries for next generation sequencing. The primer sets that target the regions of 
interest included adapter sequences for amplicon-based NGS analysis and had mean coverage of 62,000x. The PCR-products were purified using Wizard SC Gel and a PCR Clean-Up system (Promega Corporation), according to the manufacturer's protocol for targeted re-sequencing (GATC Biotech AG).

Quantification of cfDNA and cftDNA in plasma. Human telomerase reverse transcriptase (hTERT) genomic amplification was used to quantify the total amount of cfDNA using reverse transcriptase-quantitative (RT-q)PCR. To quantify cfDNA, RT-qPCR was used, based on hTERT as the target $(39,40)$. This system used two amplicon primers and a fluorgenic hybridization probe for amplifying hTERT. RT-qPCR was performed with a $20 \mu \mathrm{l}$ volume on a $7500 \mathrm{ABI}$ detection system (Applied Biosystems; Thermo Fisher Scientific, Inc.). Each run consisted of patient samples in duplicate, negative controls and a dilution of a standard TaqMan controlled human genomic DNA (Roche Diagnostics; $0.2 \mu \mathrm{g} / \mu \mathrm{l}$ ). The amount of cftDNA was calculated by multiplying the allelic fraction of the respective target gene with the total amount of cfDNA.

Analysis of serum tumor markers. Carcinoembryonic antigen (CEA), Carcinoma antigen 15-3 (CA 15-3) and carcinoma antigen 19-9 (CA 19-9) levels were analyzed during therapy at the same time-points as DNA acquisition. Analysis was performed on a Modular-E170 (CEA Ref: 11731629, CA 19-9 Ref: 11776193, CA 15-3 Ref: 03045838; Elektro Chemilumineszenz Immuno Assay; Roche Diagnostics) in cooperation with the University Institute of Medical and Chemical Laboratory Diagnostics (Salzburg, Austria).

cfDNA and cftDNA levels were correlated with CEA and CA19-9 levels in patients with colorectal and pancreatic cancer, and CA15-3 levels in breast cancer patients.

Volumetry of target lesions. In the current study, the tumor volume of two main metastatic target lesions of $5 \mathrm{CRC}$ patients was analysed in the lung and liver. For segmentation, open-radART ${ }^{\odot}$ (open-radART ion-ORAion Software Suite) was used to draw the boundaries of the tumor in each CT-slice. This segmentation produced a visual 3D-image and was used to analyse exact tumor-volume as described previously (41). Measurements of circulating cell free DNA were subsequently matched with the results of tumor-volumetry.

Statistical analysis. Statistical analysis was performed using GraphPad Prism 6.01 (GraphPad Software, Inc.) and SPSS (IBM Corporation). Correlations were analysed using the Spearmans rank test. ANOVA tests followed by post hoc Tukey tests were used to compare multiple groups. $\mathrm{P}<0.05$ was considered to indicate a statistically significant result. All error bars represent the mean \pm standard deviation.

\section{Results}

Patient characteristics and DNA isolation. A total of 15 patients were included in the current study. A total of 6 patients had metastatic pancreatic cancer, 5 patients had metastatic colorectal cancer and 4 patients had metastatic breast cancer. The median age at diagnosis was 70 years. A total of 9 patients (6 patients with PC and 3 patients with CRC) exhibited synchronous metastatic disease at diagnosis, and 6 patients developed metastasis during subsequent follow up. All patients had a median of three prior lines of palliative systemic therapy. The median overall patient survival was 93.1 weeks, from first diagnosis of metastatic disease for the whole cohort, and 183.3, 84.4 and 22.1 weeks for BC, CRC and PC, respectively. Patient characteristics are outlined in Table I.

Analysis of KRAS, NRAS, BRAF, Tp53, NOTCH, EGFR, PTEN and PI3K, revealed one common somatic mutation within Tp53, KRAS and NOTCH1 in every primary tumor sample. Five distinct mutations were revealed in KRAS, Tp53 and NOTCH1 (Fig. 1). These mutations were subsequently detected and quantified, in the respective matched plasma samples, using targeted re-sequencing. The median baseline plasma concentration of cfDNA was $340.5 \mathrm{pg} / \mu 1$ (range, 31.8-3160.8 pg/ $/ \mu \mathrm{l})$. The median concentration of cftDNA was $180.29 \mathrm{pg} / \mu \mathrm{l}$ (range, $0.011 \mathrm{pg} / \mu \mathrm{l}-1754.6 \mathrm{pg} / \mu \mathrm{l})$.

Correlation of quantitative levels of cfDNA, cftDNA and established tumor markers. cfDNA and cftDNA concentrations were compared during the course of treatment. Following analysis of all available plasma samples $(n=108)$, a modest overall correlation was observed between the amount of cfDNA and cftDNA over time (Spearman correlation coefficient 0.7536; $\mathrm{P}<0.001$; Fig. 2A).

The results of the analysis indicated that a large number of samples did not contain detectable amounts of cftDNA, while exhibiting small amounts of cfDNA. It was suggested that this may be due to a discordant expression in samples drawn in the days following treatment. We therefore focused on samples drawn at defined staging time-points.

When plasma samples, which were obtained at staging time-points were analysed, the correlation between cfDNA and cftDNA was strong (Spearman correlation coefficient 0.9221; P<0.0001; Fig. 2B). No correlation was observed between cfDNA and cftDNA in samples drawn in between staging time-points (Spearman correlation coefficient 0.0325 $\mathrm{P}=0.2113$; Fig. S1). This correlation was also demonstrated when analysing the BC (Spearman correlation coefficient 0.9335; $\mathrm{P}<0.001), \mathrm{PC}(0.9158 ; \mathrm{P}=0.002)$ and $\mathrm{CRC}(0.563$; $\mathrm{P}=0.004)$ subgroups separately (Fig. S2).

Whether the quantity of cfDNA levels of established biomarkers and if cftDNA correlated with levels of established biomarkers was assessed according to cancer subtype. In the colorectal cancer group, a significant correlation was indicated between cfDNA and CEA $(0.8962, \mathrm{P}=0.039)$ and cftDNA and CEA (0.9554; $\mathrm{P}<0.001)$.

In the PC group, a significant correlation was exhibited between cfDNA and CEA (0.8895; P=0.002; Fig. S3), but no significant correlation was observed between cftDNA and CEA (0.7235; P=0.074; Fig. S3). No correlation was indicated between cfDNA or cftDNA with CA 19-9 $(\mathrm{P}=0.192 ; \mathrm{P}=0.724$; Fig. S3).

In the $\mathrm{BC}$ group, no correlation was observed between cfDNA and CA 15-3 (0.2526, $\mathrm{P}=0.527)$ or cftDNA and CA15-3 (0.4623; P=0.702; Fig. S3).

Correlation between cfDNA, cftDNA and tumor burden. CfDNA and cftDNA concentrations and serum tumor markers were correlated with volumetric measurements of selected 
Table I. Baseline patient characteristics.

\begin{tabular}{lcc}
\hline Characteristic & Number of patients $(\mathrm{n}=15)$ & Percentage of patients $(\%)$ \\
\hline Sex & & 53.3 \\
Female & 7 & 46.7 \\
Male & $70(47-82)$ & $6(2-8)$ \\
Median age at diagnosis (years; range) & & 26.6 \\
Median follow up (months; range) & 4 \\
Cancer type & 5 \\
Breast cancer & 6 \\
Colorectal cancer & 9 & 33.3 \\
Pancreatic cancer & $3(1-6)$ \\
Primary metastatic disease & $93.1(15.8-196.9)$ \\
Median prior lines of palliative therapy (range) & $183.3(149.9-196.2)$ & 60.1 \\
Median overall survival (range; weeks) & $84.4(55.5-187.3)$ & \\
Breast cancer & $22.1(12.3-63.9)$ & \\
Colorectal cancer & & \\
Pancreatic cancer &
\end{tabular}

$\mathrm{BC}$, breast cancer; $\mathrm{CRC}$, colorectal cancer; PC, pancreatic cancer.
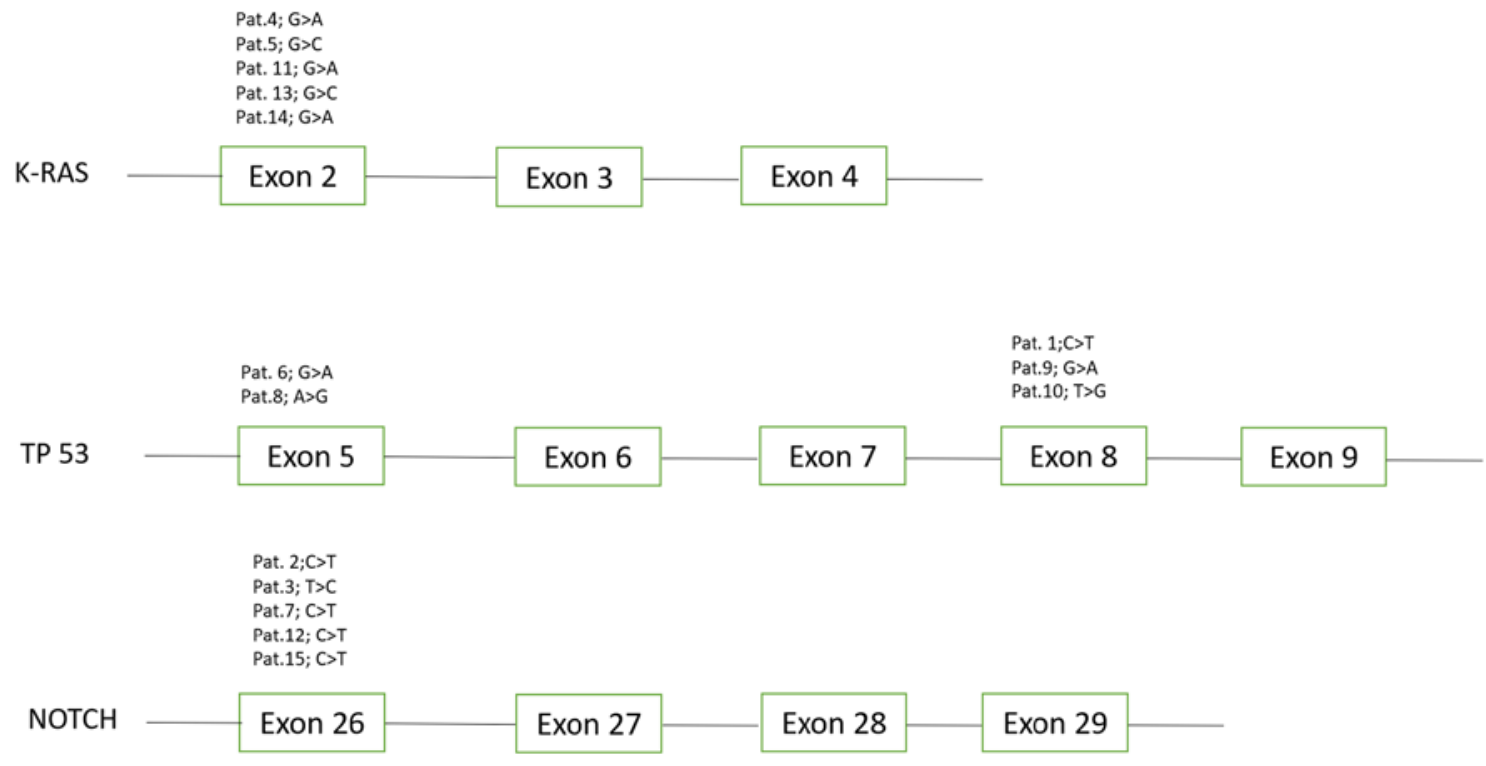

Figure 1. Overview of all detected mutations in the primary tumor sample. Mutations were identified in KRAS (5 patients), Tp53 (5 patients) and NOTCH 2 (5 patients).

metastatic target lesions in the liver and the lung of five patients with metastatic CRC. A significant correlation was demonstrated between tumor volume in the liver and cfDNA $(\mathrm{P}=0.016)$, and tumor volume in the lung and cfDNA $(\mathrm{P}=0.003)$.

The results of cftDNA and tumor volume analysis revealed a borderline significant correlation between tumor burden in the liver $(\mathrm{P}=0.058)$, and no correlation in the lung $(\mathrm{P}=0.383)$.

The results of the comparison of tumor marker levels of CA 19-9 and CEA with tumor volume, no correlation was indicated between tumor burden in the liver $(\mathrm{P}=0.104$ for $\mathrm{CA} 19-9$; $\mathrm{P}=0.873$ for $\mathrm{CEA})$ or the lung $(\mathrm{P}=0.789$ for $\mathrm{CA} 19-9 ; \mathrm{P}=0.052$ for CEA).
cfDNA, cftDNA and clinical response. The current study investigated how changes in cfDNA or cftDNA during treatment correlated with the response to therapy (assessed via imaging), and how cfDNA and cftDNA performed compared to currently used clinical biomarkers. Therefore, disease response assessed by $\mathrm{CT}$ imaging [partial response (PR), stable disease (SD) or progressive disease (PD) according to RECIST] was compared with concentration changes of cfDNA and cftDNA and tumor markers over time. The ratio of cf (t) DNA and tumor markers before and at the time of the respective staging $\mathrm{CT}$ was measured (i.e. $\mathrm{cfDNA}_{\text {before staging }}$ $\left.(\mathrm{pg} / \mu \mathrm{l}) / \mathrm{cfDNA}_{\text {staging }}(\mathrm{pg} / \mu \mathrm{l})\right)$, and this ratio was correlated with 
A

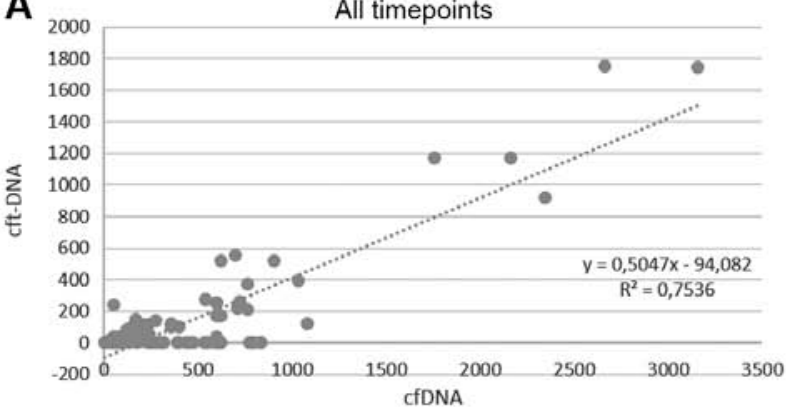

B

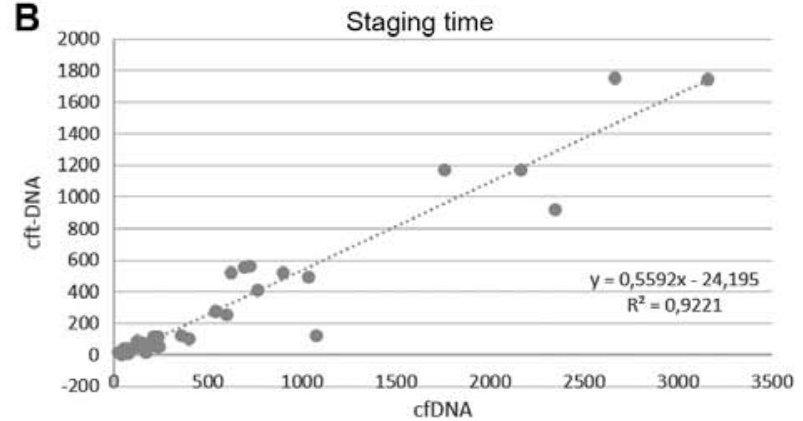

Figure 2. (A) Correlation between cfDNA levels (pg/ $\mu$ l) and cftDNA levels (pg/ $/ \mu \mathrm{l})$ for all available samples (n=108). (B) Correlation between cfDNA and cftDNA for samples obtained at staging time-points $(\mathrm{n}=38)$. cfDNA, circulating cell-free DNA; cftDNA, circulating cell-free tumor DNA.

the imaging result. A significant correlation was exhibited between the response assessed via imaging, and cfDNA and cftDNA (Fig. 3A). However, no correlation was observed between imaging results and tumor marker changes (Fig. 3B).

Subsequently, whether early changes in the ratio of cfDNA to cftDNA could predict treatment response was assessed. The ratio of cfDNA to cftDNA at time-points between treatment start and the first restaging CT were compared. However, the results did not demonstrate a significant correlation between treatment response and changes to the cfDNA/cftDNA ratio before imaging (Fig. S4). However, there was a marked trend, which was not statistically significant.

\section{Discussion}

In the current study, the potential role of cfDNA as a quantitative monitoring tool during cancer therapy in daily clinical practice was explored, and cfDNA was compared between imaging techniques and classical tumor markers that are currently used.

A total of 15 patients with three common tumor subtypes were assessed. The majority of patients had metastatic disease at diagnosis and the median OS observed in the respective cancer subtypes was in line with previously published cohorts (42-46).

Analyses were performed using mutations in commonly mutated genes, which were indicated by previously published data $(21,36,47-54)$. The results of Sanger sequencing showed the presence of mutations within KRAS, Tp53 and NOTCH1 in the primary tumor sample. No other mutations were investigated. The overall concentration of cfDNA in our cohort was comparable to previously published reports $(21,24,55)$.

A strong correlation was demonstrated between cfDNA and cftDNA from plasma samples obtained at staging timepoints, compared to the correlation in all plasma samples or samples drawn in-between staging time-points.

In our practice, restaging time-points were often scheduled two to three weeks following the last application of systemic therapy (prior to the next scheduled application). Therefore, less fluctuations in cfDNA or cftDNA levels at these time-points were expected, presumably due to less tumor cell turnover. The data revealed that the time-point of sample acquisition was important for the interpretation of cfDNA or cftDNA levels, and should be further standardized in the future.

In contrast, it was observed that the changes in ratio between cfDNA and cftDNA in-between staging CTs may be able to predict treatment response. However, due to the small sample size we were only able to see a trend, which was not statistically significant and therefore needs further investigation in future trials.

cfDNA was subsequently compared with tumor markers in colorectal cancer, and a correlation was indicated between CEA, but not CA 19-9. These results may be due to CEA being a more specific tumor marker in CRC than CA 19-9 (56-60).

In the pancreatic cancer group, a correlation was indicated between cfDNA and CEA, but not CA 19-9. A rise in cfDNA was observed when patients were examined in more detail, which correlated with disease progression upon imaging, but was not reflected by a rise in CA 19-9. Likewise, no correlation was demonstrated between cfDNA/cftDNA and CA 15-3 in the breast cancer group. However, a rise in cfDNA/cftDNA correlated with disease progression upon imaging, but was not reflected by a rise in CA 15-3. These observations support the potentially superior reflection of tumor dynamics with the use of cfDNA and cftDNA compared to classical biomarkers.

The results of the comparison of treatment response upon imaging demonstrated a stronger correlation between clinical staging and cfDNA and cftDNA than between classical tumor markers, further highlighting the potential of this new biomarker.

In the current study, tumor volumetric measurements were also compared during treatment with cfDNA/cftDNA, in comparison with classical biomarkers. A total of 5 patients with CRC who all had metastatic disease in the lung or the liver were assessed. These 5 patients were focused on due to the fact that volumetry of metastatic lesions can be performed more accurately in the lung and liver because of the better contrast between tumor and normal organ tissue. A strong correlation was observed between the amount of cfDNA and volume of the metastatic lesions. This correlation could not be demonstrated with classical biomarkers. cfDNA indicated a stronger correlation with the metastatic tumor burden than cftDNA. Possible explanations for this observation are the molecular heterogeneity of the tumor, clonal evolution during treatment or changes in the genetic background of the tumor, which were not detected by targeted resequencing.

The sample size of 15 patients in this study is relatively small, therefore further trials with higher patient numbers are required to confirm the reported findings. However, the correlation between cfDNA and cftDNA and the correlation 
A

Ratio cft-DNA

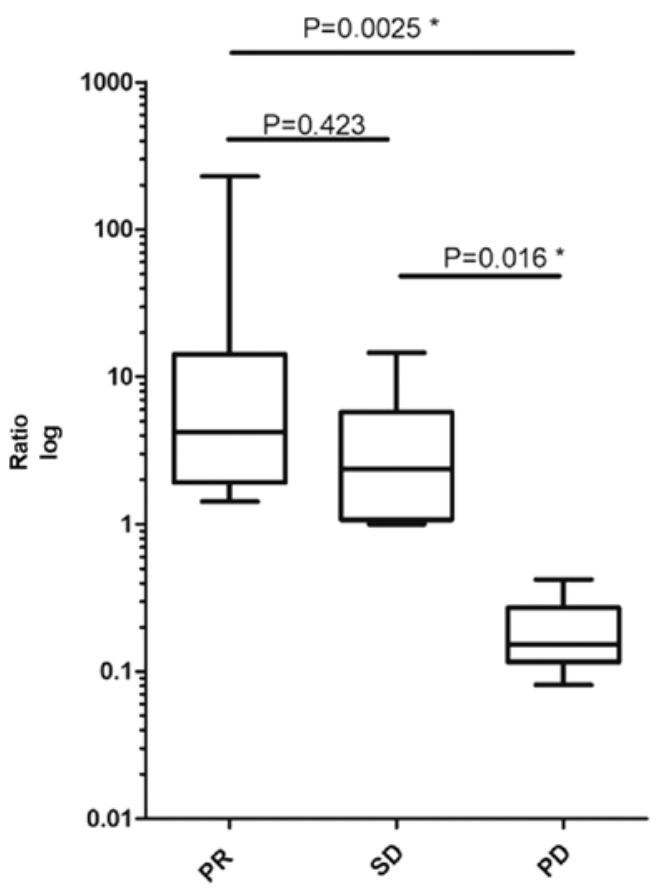

B

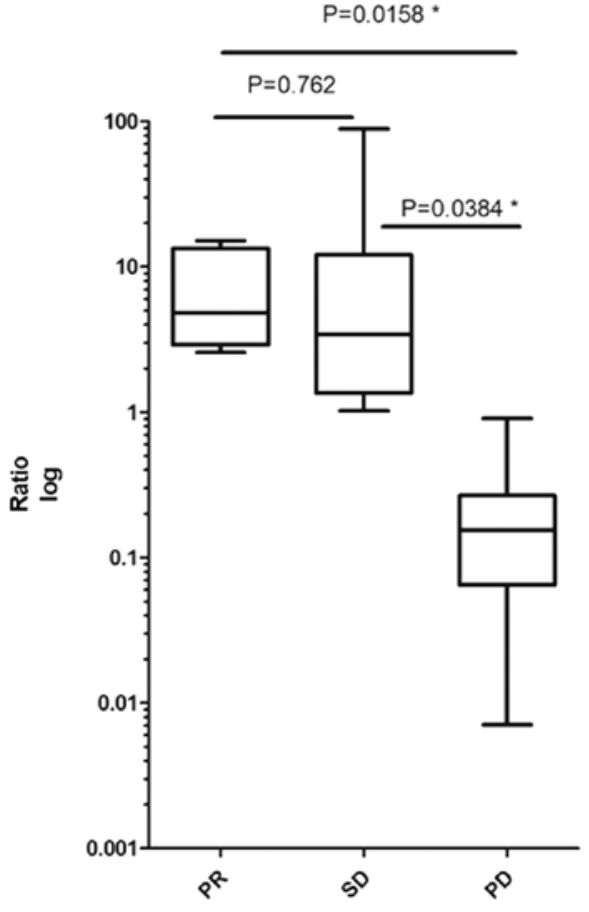

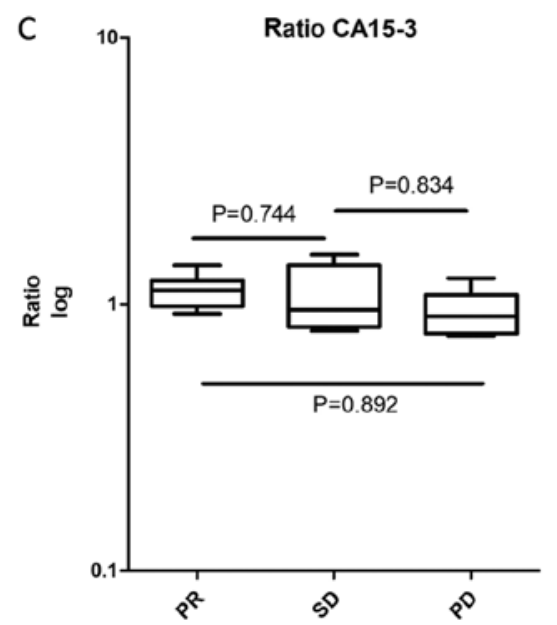
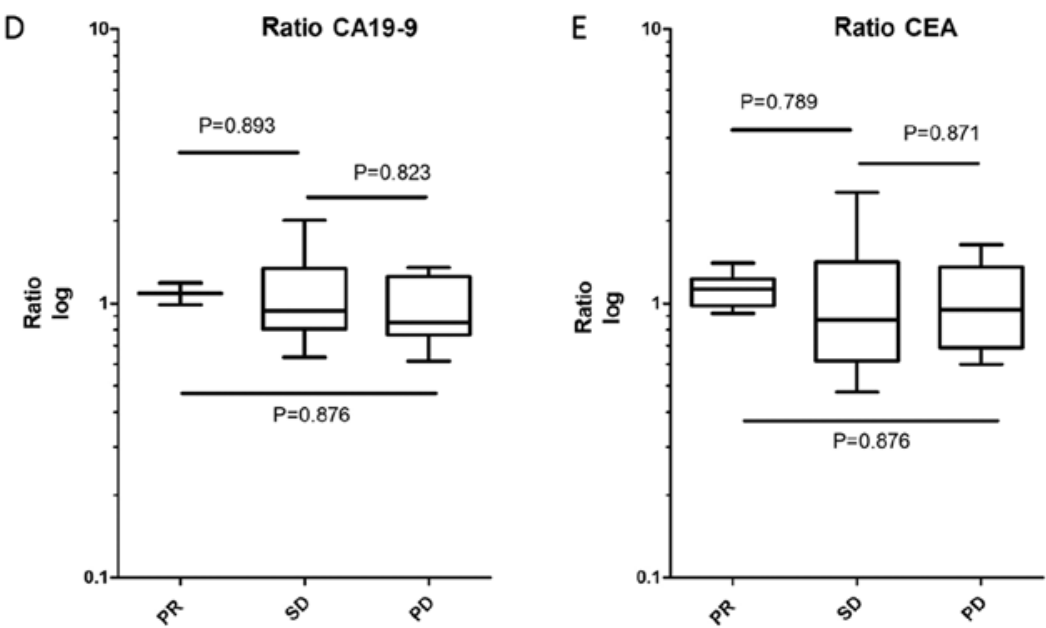

Figure 3. (A) Ratio of cfDNA before staging and cfDNA staging plotted against treatment response according to RECIST. (B) Ratio of cftDNA before staging and cftDNA staging blotted against treatment response according to RECIST. (C) Ratio of CA15-3 before staging and CA15-3 staging blotted against treatment response according to RECIST. (D) Ratio of CA19-9 before staging and CA19-9 staging plotted against treatment response according to RECIST. (E) Ratio of CEA before staging and CEA staging blotted against treatment response according to RECIST. cfDNA, circulating cell-free DNA; cftDNA, circulating cell-free tumor DNA; RECIST, Response Evaluation Criteria in Solid Tumors; CEA, Carcinoembryonic antigen; CA15-3, Carcinoma Antigen 15-3; CA19-9, Carcinoma Antigen 19-9.

between clinical staging and cfDNA/cftDNA in our study is significant despite the small patient number. Patients were selected with three different tumor types, which allowed investigation across different disease entities; however, this leads to a certain amount of heterogeneity of the data. Tumor volumetry was only available for the CRC group, so conclusions regarding the other two tumor types could not be made. We were not able to perform a fragment analysis of cfDNA due to the low concentration in the plasma samples. This should be implemented in future studies.

Overall, the results of the current study indicated that cfDNA and cftDNA outperformed currently used biomarkers in predicting the response to therapy and quantifying tumor burden in a small patient cohort. Standards for the optimal time-point of sample acquisition for cfDNA analysis should be defined further in the future.

\section{Acknowledgements}

The authors would like to thank Mrs Ulrike Seidl, Mrs Manuela Glück and Mrs Maria Dreier, (IIIrd Medical Department with Hematology and Medical Oncology, Oncologic Center, Paracelsus Medical University Salzburg), for sample collection. 


\section{Funding}

This work was supported by the Österreichische Krebshilfe Salzburg.

\section{Availability of data and materials}

The datasets used and/or analysed during the current study are available from the corresponding author on reasonable request.

\section{Authors' contributions}

$\mathrm{CH}, \mathrm{ML}$ and AE: Data analysis, review and writing. $\mathrm{CH}$ : Molecular analysis. CH, ML, LW and TMel: Data analysis, MM, DA, RG, GR and DN: Statistical analysis and interpretation, PS and TMei CT/PET-CT analysis, AE and RG supervised this work and assisted in preparing the manuscript. All authors have read and approved the final manuscript. All co-authors provided continuous intellectual guidance, repeatedly reviewed and edited the manuscript, and gave the final approval for submission.

\section{Ethics approval and consent to participate}

The current study was approved by the local institutional research ethics committee (permit no. 415-E/1469/11-2013) and all patients provided written informed consent prior to blood or tissue sampling.

\section{Patient consent for publication}

Not applicable.

\section{Competing interests}

The authors declare that they have no competing interests.

\section{References}

1. Ciombor KK, Haraldsdottir S and Goldberg RM: How can next-generation sequencing (genomics) help us in treating colorectal cancer? Curr Colorectal Cancer Rep 10: 372-379, 2014

2. Hanahan D and Weinberg RA: Hallmarks of cancer: The next generation. Cell 144: 646-674, 2011.

3. Diaz LA Jr and Bardelli A: Liquid biopsies: Genotyping circulating tumor DNA. J Clin Oncol 32: 579-586, 2014.

4. Choi JH, Ahn MJ, Rhim HC, Kim JW, Lee GH, Lee YY and Kim IS: Comparison of WHO and RECIST criteria for response in metastatic colorectal carcinoma. Cancer Res Treat 37: 290-293, 2005.

5. Padhani AR and Ollivier L: The RECIST (Response Evaluation Criteria in Solid Tumors) criteria: Implications for diagnostic radiologists. Br J Radiol 74: 983-986, 2001.

6. Therasse P, Le Cesne A, Van Glabbeke M, Verweij J and Judson I; EORTC Soft Tissue and Bone SarcomaGroup: RECIST vs. WHO: Prospective comparison of response criteria in an EORTC phase II clinical trial investigating ET-743 in advanced soft tissue sarcoma. Eur J Cancer 41: 1426-1430, 2005.

7. Watanabe H, Yamamoto S, Kunitoh H, Sekine I, Yamamoto N, Ohe Y, Tamura T, Kodama T, Sugimura K and Saijo N: Tumor response to chemotherapy: The validity and reproducibility of RECIST guidelines in NSCLC patients. Cancer Sci 94: 1015-1020, 2003.

8. Harris L, Fritsche H, Mennel R, Norton L, Ravdin P, Taube S, Somerfield MR, Hayes DF and Bast RC Jr; American Society of Clinical Oncology: American Society of Clinical Oncology 2007 update of recommendations for the use of tumor markers in breast cancer. J Clin Oncol 25: 5287-5312, 2007.
9. Schwartz LH, Seymour L, Litière S, Ford R, Gwyther S, Mandrekar S, Shankar L, Bogaerts J, Chen A, Dancey J, et al: RECIST 1.1-Standardisation and disease-specific adaptations: Perspectives from the RECIST Working Group. Eur J Cancer 62: 138-145, 2016.

10. Arrieta O, Villarreal-Garza C, Martínez-Barrera L, Morales M, Dorantes-Gallareta Y, Peña-Curiel O, Contreras-Reyes S, Macedo-Pérez EO and Alatorre-Alexander J: Usefulness of serum carcinoembryonic antigen (CEA) in evaluating response to chemotherapy in patients with advanced non small-cell lung cancer: A prospective cohort study. BMC Cancer 13: 254, 2013.

11. Ballehaninna UK and Chamberlain RS: The clinical utility of serum CA 19-9 in the diagnosis, prognosis and management of pancreatic adenocarcinoma: An evidence based appraisal. J Gastrointest Oncol 3: 105-119, 2012.

12. O'Connell M: PET-CT modification of RECIST guidelines. J Natl Cancer Inst 96: 801-802; author reply 802, 2004.

13. Watanabe S, Nakamura Y, Kariatsumari K, Nagata T, Sakata R, Zinnouchi S and Date K: Pulmonary paragonimiasis mimicking lung cancer on FDG-PET imaging. Anticancer Res 23: 3437-3440, 2003.

14. Solinas C, Porcu M, Hlavata Z, De Silva P, Puzzoni M, Willard-Gallo K, Scartozzi M and Saba L: Critical features and challenges associated with imaging in patients undergoing cancer immunotherapy. Crit Rev Oncol Hematol 120: 13-21, 2017.

15. Kidess E and Jeffrey SS: Circulating tumor cells versus tumor-derived cell-free DNA: Rivals or partners in cancer care in the era of single-cell analysis? Genome Med 5: 70, 2013.

16. Alix-Panabières $C$ and Pantel $\mathrm{K}$ : Challenges in circulating tumour cell research. Nat Rev Cancer 14: 623-631, 2014.

17. Dive $C$ and Brady G: SnapShot: Circulating tumor cells. Cell 168: 742-742.e1, 2017.

18. Pantel K and Alix-Panabières C: Liquid biopsy in 2016: Circulating tumour cells and cell-free DNA in gastrointestinal cancer. Nat Rev Gastroenterol Hepatol 14: 73-74, 2017.

19. González-Masiá JA, García-Olmo D and García-Olmo DC: Circulating nucleic acids in plasma and serum (CNAPS): Applications in oncology. Onco Targets Ther 6: 819-832, 2013.

20. Circulating nucleic acids in plasma/serum III and serum proteomics. Proceedings of the Third International Symposium. November 9-12, 2003. Santa Monica, California, USA. Ann NY Acad Sci 1022: 1-322, 2004.

21. Anker P, Lyautey J, Lederrey C and Stroun M: Circulating nucleic acids in plasma or serum. Clin Chim Acta 313: 143-146, 2001.

22. Anker P, Mulcahy $\mathrm{H}$ and Stroun M: Circulating nucleic acids in plasma and serum as a noninvasive investigation for cancer: Time for large-scale clinical studies? Int J Cancer 103: 149-152, 2003.

23. Diehl F, Schmidt K, Choti MA, Romans K, Goodman S, Li M, Thornton K, Agrawal N, Sokoll L, Szabo SA, et al: Circulating mutant DNA to assess tumor dynamics. Nat Med 14: 985-990, 2008.

24. Fleischhacker M and Schmidt B: Free circulating nucleic acids in plasma and serum (CNAPS)-useful for the detection of lung cancer patients? Cancer Biomark 6: 211-219, 2010.

25. Gahan PB: Circulating nucleic acids in plasma and serum: Roles in diagnosis and prognosis in diabetes and cancer. Infect Disord Drug Targets 8: 100-108, 2008.

26. Rolfo C, Castiglia M, Hong D, Alessandro R, Mertens I, Baggerman G, Zwaenepoel K, Gil-Bazo I, Passiglia F, Carreca AP, et al: Liquid biopsies in lung cancer: The new ambrosia of researchers. Biochim Biophys Acta 1846: 539-546, 2014.

27. Taback B and Hoon DS: Circulating nucleic acids in plasma and serum: Past, present and future. Curr Opin Mol Ther 6: 273-278, 2004.

28. Tsang JC and Lo YM: Circulating nucleic acids in plasma/serum. Pathology 39: 197-207, 2007.

29. Yong E: Cancer biomarkers: Written in blood. Nature 511: 524-526, 2014.

30. Cervera Deval J: RECIST and the radiologist. Radiologia 56: 193-205, 2014 (In Spanish).

31. Krajewski KM, Nishino M, Ramaiya NH and Choueiri TK: RECIST 1.1 compared with RECIST 1.0 in patients with advanced renal cell carcinoma receiving vascular endothelial growth factor-targeted therapy. AJR Am J Roentgenol 204: W282-W288, 2015.

32. Lencioni R and Llovet JM: Modified RECIST (mRECIST) assessment for hepatocellular carcinoma. Semin Liver Dis 30: 52-60, 2010.

33. van Persijn van Meerten EL, Gelderblom H and Bloem JL: RECIST revised: Implications for the radiologist. A review article on the modified RECIST guideline. Eur Radiol 20: 1456-1467, 2010 
34. Lemoine NR: Molecular advances in pancreatic cancer. Digestion 58: 550-556, 1997.

35. Oden-Gangloff A, Di Fiore F, Bibeau F, Lamy A, Bougeard G, Charbonnier F, Blanchard F, Tougeron D, Ychou M, Boissière $\mathrm{F}$, et al: TP53 mutations predict disease control in metastatic colorectal cancer treated with cetuximab-based chemotherapy. Br J Cancer 100: 1330-1335, 2009.

36. Olivier M, Hollstein M and Hainaut P: TP53 mutations in human cancers: Origins, consequences, and clinical use. Cold Spring Harb Perspect Biol 2: a001008, 2010.

37. Shaw JA, Page K, Blighe K, Hava N, Guttery D, Ward B, Brown J, Ruangpratheep C, Stebbing J, Payne R, et al: Genomic analysis of circulating cell-free DNA infers breast cancer dormancy. Genome Res 22: 220-231, 2012.

38. Hufnagl C, Stöcher M, Moik M, Geisberger R and Greil R: A modified Phenol-chloroform extraction method for isolating circulating cell free DNA of tumor patients. J Nucleic Acids Invest 4, 2013.

39. Paci M, Maramotti S, Bellesia E, Formisano D, Albertazzi L, Ricchetti T, Ferrari G, Annessi V, Lasagni D, Carbonelli C, et al: Circulating plasma DNA as diagnostic biomarker in non-small cell lung cancer. Lung Cancer 64: 92-97, 2009.

40. Sirera R, Bremnes RM, Cabrera A, Jantus-Lewintre E, Sanmartín E, Blasco A, Del Pozo N, Rosell R, Guijarro R, Galbis J, et al: Circulating DNA is a useful prognostic factor in patients with advanced non-small cell lung cancer. J Thorac Oncol 6: 286-290, 2011.

41. Marten K, Auer F, Schmidt S, Rummeny EJ and Engelke C: Automated CT volumetry of pulmonary metastases: The effect of a reduced growth threshold and target lesion number on the reliability of therapy response assessment using RECIST criteria. Eur Radiol 17: 2561-2571, 2007.

42. Ferrero A, Bernad B, Campos J, Perales E, Velázquez JL and Martínez-Verdú FM: Color characterization of coatings with diffraction pigments. J Opt Soc Am A Opt Image Sci Vis 33: 1978-1988, 2016.

43. Gordis L and Gold EB: Epidemiology of pancreatic cancer. World J Surg 8: 808-821, 1984

44. Maughan NJ and Quirke P: Genomics in colorectal cancer: Godsend or gimmick? Scand J Gastroenterol (Suppl): 26-29, 2003

45. Michaud DS: Epidemiology of pancreatic cancer. Minerva Chir 59: 99-111, 2004

46. Stein RG, Wollschläger D, Kreienberg R, Janni W, Wischnewsky M, Diessner J, Stüber T, Bartmann C, Krockenberger M, Wischhusen J, et al: The impact of breast cancer biological subtyping on tumor size assessment by ultrasound and mammography-a retrospective multicenter cohort study of 6543 primary breast cancer patients. BMC Cancer 16: 459, 2016.

47. Anker P, Lefort F, Vasioukhin V, Lyautey J, Lederrey C, Chen XQ, Stroun M, Mulcahy HE and Farthing MJ: K-ras mutations are found in DNA extracted from the plasma of patients with colorectal cancer. Gastroenterology 112: 1114-1120, 1997.

48. Boeck S, Jung A, Laubender RP, Neumann J, Egg R, Goritschan C, Vehling-Kaiser U, Winkelmann C, Fischer von Weikersthal L, Clemens MR, et al: EGFR pathway biomarkers in erlotinib-treated patients with advanced pancreatic cancer: Translational results from the randomised, crossover phase 3 trial AIO-PK0104. Br J Cancer 108: 469-476, 2012.
49. Chappell WH, Steelman LS, Long JM, Kempf RC, Abrams SL Franklin RA, Bäsecke J, Stivala F, Donia M, Fagone P, et al: Ras/Raf/MEK/ERK and PI3K/PTEN/Akt/mTOR inhibitors: Rationale and importance to inhibiting these pathways in human health. Oncotarget 2: 135-164, 2011.

50. De Luca A, Maiello MR, D'Alessio A, Pergameno $M$ and Normanno N: The RAS/RAF/MEK/ERK and the PI3K/AKT signalling pathways: Role in cancer pathogenesis and implications for therapeutic approaches. Expert Opin Ther Targets 16 (Suppl 2): S17-S27, 2012.

51. Deramaudt T and Rustgi AK: Mutant KRAS in the initiation of pancreatic cancer. Biochim Biophys Acta 1756: 97-101, 2005.

52. Lui VW, Hedberg ML, Li H, Vangara BS, Pendleton K, Zeng Y, Lu Y, Zhang Q, Du Y, Gilbert BR, et al: Frequent mutation of the PI3K pathway in head and neck cancer defines predictive biomarkers. Cancer Discov 3: 761-769, 2013.

53. Rao SS, O'Neil J, Liberator CD, Hardwick JS, Dai X, Zhang T, Tyminski E, Yuan J, Kohl NE, Richon VM, et al: Inhibition of NOTCH signaling by gamma secretase inhibitor engages the RB pathway and elicits cell cycle exit in T-cell acute lymphoblastic leukemia cells. Cancer Res 69: 3060-3068, 2009.

54. van Krieken JH, Jung A, Kirchner T, Carneiro F, Seruca R, Bosman FT, Quirke P, Fléjou JF, Plato Hansen T, de Hertogh G, et al: KRAS mutation testing for predicting response to anti-EGFR therapy for colorectal carcinoma: Proposal for an European quality assurance program. Virchows Arch 453: 417-431, 2008.

55. Heitzer E, Auer M, Ulz P, Geigl JB and Speicher MR: Circulating tumor cells and DNA as liquid biopsies. Genome Med 5: 73, 2013.

56. Estakhri R, Ghahramanzade A, Vahedi A and Nourazarian A: Serum levels of CA15-3, AFP, CA19-9 and CEA tumor markers in cancer care and treatment of patients with impaired renal function on hemodialysis. Asian Pac J Cancer Prev 14: 1597-1599, 2013.

57. Guo Q, Kang M, Zhang B, Chen Y, Dong X and Wu Y: Elevated levels of CA 19-9 and CEA in pancreatic cancer-associated diabetes. J Cancer Res Clin Oncol 136: 1627-1631, 2010.

58. Hegele A, Mecklenburg V, Varga Z, Olbert P, Hofmann R and Barth P: CA19.9 and CEA in transitional cell carcinoma of the bladder: Serological and immunohistochemical findings. Anticancer Res 30: 5195-5200, 2010.

59. Ince AT, Yıldız K, Baysal B, Danalığlu A, Kocaman O, Tozlu M, Gangarapu V, Sarbay Kemik A, Uysal Ö and Sentürk H: Roles of serum and biliary CEA, CA19-9, VEGFR3, and TAC in differentiating between malignant and benign biliary obstructions. Turk J Gastroenterol 25: 162-169, 2014.

60. Thomakos N, Rodolakis A, Zagouri F, Zacharakis D, Sotiropoulou M, Akrivos N, Haidopoulos D, Papadimitriou CA Dimopoulos MA and Antsaklis A: Serum CA 125, CA 15-3, CEA, and CA 19-9: A prognostic factor for uterine carcinosarcomas? Arch Gynecol Obstet 287: 97-102, 2013. 